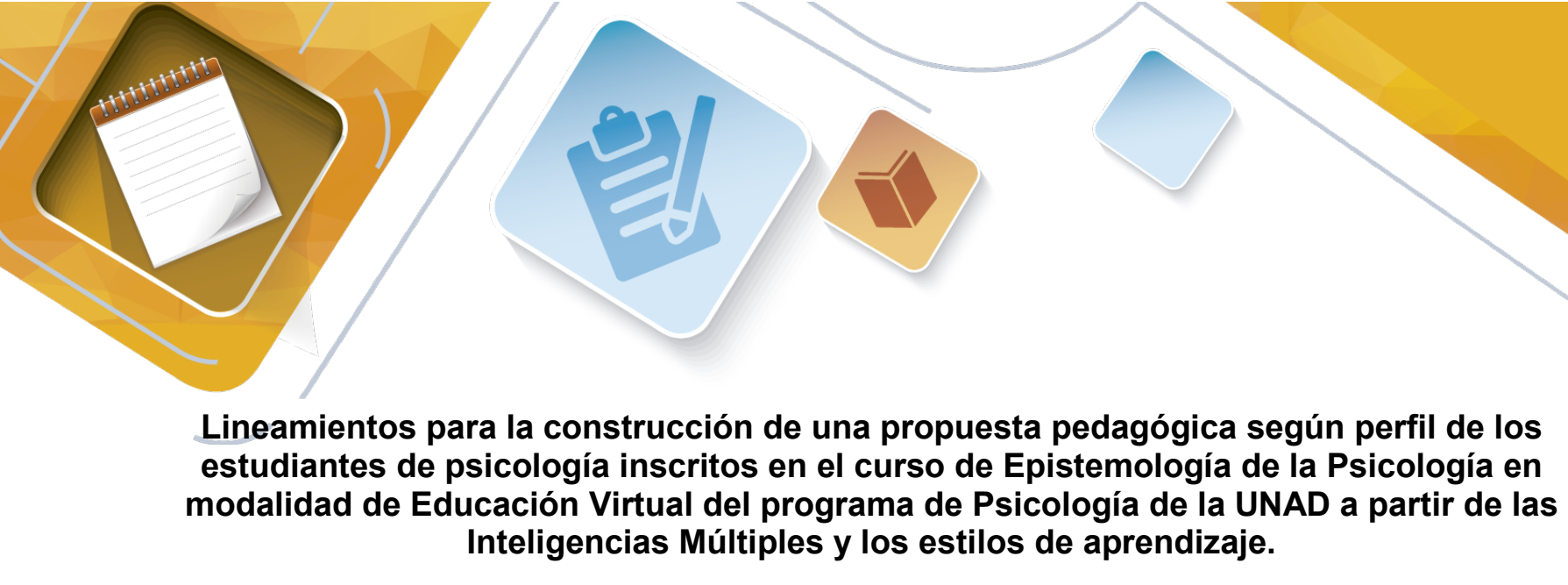

\title{
Lineamientos para la construcción de una propuesta pedagógica según perfil de los estudiantes de psicología inscritos en el curso de Epistemología de la Psicología en modalidad de Educación Virtual del programa de Psicología de la UNAD a partir de las Inteligencias Múltiples y los estilos de aprendizaje.
}

Claudia Andrea Paredes Clara Tatiana Verney Marcela Granada Laura Gisella Tolosa

Con la finalidad de proponer una estrategia pedagógica que favorezca el aprendizaje de los estudiantes que inicia en el programa de psicología en la UNAD, se hace necesario reconocer la importancia del funcionamiento del cerebro, del sistema nervioso y de su plasticidad, ya que ésta información aclara la comprensión de cómo se potencia el aprendizaje matizado por un componente cultural. (Gardner,1994).

En este sentido, se considera relevante incorporar, en la labor educativa, el aporte que las neurociencias realizan para la comprensión de cómo funciona el cerebro en el acto educativo y así proponer estrategias que dinamicen el aprendizaje de cada uno de los estudiantes que ingresa al programa de Psicología. Por ello, se realizó un estudio con 107 estudiantes inscritos en los periodos 16-1 y 16-2 del 2018, en el cual se exploraron las inteligencias múltiples desde la propuesta de Gadner y las dominancias cerebrales desde la teoría de los hemisferios cerebrales de Ned Hermann encontrando que la inteligencia múltiple predomínate en este grupo fue la inteligencia intrapersonal y el cuadrante dominante fue el B-C. Sin embargo, el estudio evidencia que cada una de las inteligencias y de los cuadrantes se encuentran presentes en los estudiantes. (Paredes, et. Al., 2018)

Estos resultados, llevan a la reflexión desde la teoría de las inteligencias múltiples de Gardner (1994) que enfatiza en la naturaleza dinámica del intelecto y la inteligencia como el conjunto de habilidades que pueden ser perfeccionadas con la experiencia en el que el contexto estimula desarrollo de las diferentes habilidades que las integran. En otras palabras, la inteligencias que se consideran potenciales, son una interacción entre la biología y las oportunidades de aprendizaje que pueden existir en una cultura. (Gardner, 1983/2005, 2005, p. 289).

Por otra parte, la teoría de cerebro total de Hermann, en la que se establece que cada uno de los cuadrantes domina funciones particulares y en la que se considera que los estilos de aprendizaje son la forma en que cada persona se enfrenta a las tareas que requieren aprendizaje las cuales son constantes en el tiempo, siendo resultado de la influencia de la herencia, experiencias de cada individuo y las exigencias del contexto. De igual manera, se reconoce que cada persona desarrolla una dominancia cerebral que permite procesar la información que percibe del entorno direccionando sus habilidades las cuales favorecen un tipo de pensamiento ya sea realista, idealista, pragmático o instintivo según lo propuesto por Hermann. A continuación, la figura 1 , explica cada uno de los cuadrantes.

Figura1.Cudrantes cerebrales según Ned Hermann. 


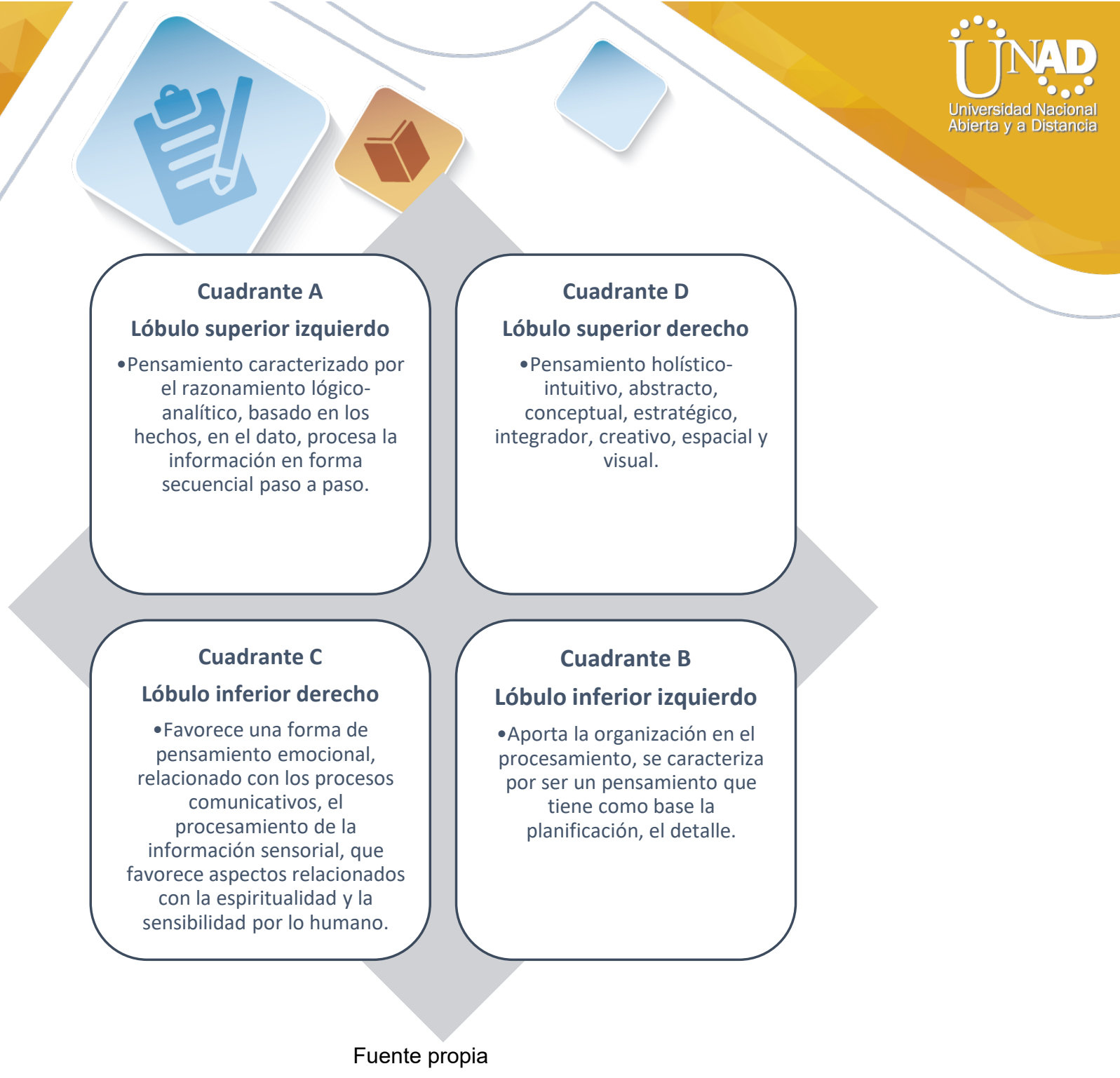

Con base en lo anterior, se hace necesario que se combinen las habilidades y tipos de pensamiento de cada hemisferio con la finalidad que se produzca un aprendizaje eficiente ya que posibilitan un desarrollo integral, desde los racional, lo emocional, lo creativo y lo práctico en cada persona. Adicionalmente, desde el componente biológico, se puede concluir que cada persona nace con una dominancia cerebral. Es decir, una predisposición para utilizar un hemisferio con sus características particulares, para interactuar con su medio (Salas et al, 2004).

Desde lo expuesto, la propuesta pedagógica, debe incorporar proyectos que faciliten todos los tipos de inteligencia que actúen de forma integral con cada una de las inteligencias y los dos hemisferios cerebrales y a su vez, que lo aprendido, se encuentre conectado con las experiencias de los estudiantes con el contexto, ya que, de esta manera, se favorece el aprendizaje significativo.

En este sentido, las didácticas requieren incluir en su elaboración medios, lógicas y propuestas versátiles que permitan que los contenidos puedan ser personalizados dependiendo del tipo e inteligencia y estilo de aprendizaje que utilice el estudiante de manera que se incluyan en formatos que contemplen la vía preferida perceptiva (Visual, auditiva, texto) de base para presentar las orientaciones que se brinde a lo largo del curso y de igual forma los contenidos y opciones para el desarrollo de actividades y evaluación. 
Por su versatilidad son herramientas esenciales para potenciar los estilos de aprendizajepensamiento e inteligencia ya que oscilan entre programas básicos de texto a simuladores que permiten crear ambientes que permiten practicas especializadas de laboratorio, acercando y creando otras formas de realidad.

En este contexto también son recursos potentes, las comunidades virtuales para proponer nuevas formas de interacción a través de redes sociales; la posibilidad de comunicación con diversas áreas geográficas del mundo, permite generar nuevos espacios educativos e interculturales y colectivos sociales.

De este modo, se puede pensar en potenciar nuevas posibilidades de comunicación y de reconocimiento para emergentes formas de razonamiento colectivo, sobre fenómenos sociales, que influyan en las dinámicas subjetivas, de grupos e individuos, derivando en nuevas formas de apropiación de lo que es humano.

Estas reflexiones ante el acto educativo desarrolladas como base de esta investigación, pretenden se aportar en el mejoramiento del proceso formativo del estudiante atendiendo la diversidad de la complejidad humana desde los procesos de aprendizaje, creando en las propuestas pedagógicas rutas o vías que posibiliten opciones de expresión de los diversos estilos de aprendizaje- formas de pensamiento y desarrollo de las inteligencias múltiples.

Para ello, es necesario reconocer y trabajar las variables implicadas en el proceso, es decir las relacionadas con el estudiante, docente; la pedagogía y didáctica y las variables del entorno, de manera que favorezca un aprendizaje personalizado, que estudie cada aspecto en la planeación del acto educativo. 